FACTA UNIVERSITATIS (NIŠ)

Ser. Math. Inform. Vol. 35, No 2 (2020), 321-332

https://doi.org/10.22190/FUMI2002321Y

\title{
PSEUDO-PARALLEL KAEHLERIAN SUBMANIFOLDS IN COMPLEX SPACE FORMS
}

\begin{abstract}
Ahmet Yildiz
(C) 2020 by University of Niš, Serbia | Creative Commons Licence: CC BY-NC-ND

Abstract. Let $\tilde{M}^{m}(c)$ be a complex $m$-dimensional space form of holomorphic sectional curvature $c$ and $M^{n}$ be a complex $n$-dimensional Kaehlerian submanifold of $\tilde{M}^{m}(c)$. We prove that if $M^{n}$ is pseudo-parallel and $L n-\frac{1}{2}(n+2) c \geqslant 0$ then $M^{n}$ is totally geodesic. Also, we study Kaehlerian submanifolds of complex space form with recurrent second fundamental form.

Keywords. Pseudo-parallel submanifolds; Kaehlerian submanifolds; recurrent second fundamental form.
\end{abstract}

\section{Introduction}

Among all submanifolds of an almost Hermitian manifold, there are two typical classes: one is the class of holomorphic submanifolds and the other is the class of totally real submanifolds. A submanifold $M$ of an almost Hermitian manifold $\tilde{M}$ is called holomorphic (resp. totally real) if each tangent space of $M$ is mapped into itself (resp. the normal space) by the almost complex structure of $\tilde{M}$. There are many results in the theory of holomorphic submanifolds.

The class of isometric immersions in a Riemannian manifold with parallel second fundamental form is very wide, as it is shown, for instance, in the classical Ferus paper [10]. Certain generalizations of these immersions have been studied, obtaining classification theorems in some cases.

Given an isometric immersion $f: M \longrightarrow \tilde{M}$, let $h$ be the second fundamental form and $\bar{\nabla}$ the van der Waerden-Bortolotti connection of $M$. Then J. Deprez defined the immersion to be semi-parallel if

$$
\bar{R}(X, Y) \cdot h=\left(\bar{\nabla}_{X} \bar{\nabla}_{Y}-\bar{\nabla}_{Y} \bar{\nabla}_{X}-\bar{\nabla}_{[X, Y]}\right) h=0,
$$

Received February 02, 2019; accepted March 17, 2019

2010 Mathematics Subject Classification. Primary 53B20; Secondary 53B25, 53B50, 53C15 
holds for any vector fields $X, Y$ tangent to $M$. J. Deprez mainly paid attention to the case of semi-parallel immersions in real space forms (see [5] and [6]). Later, Ü. Lumiste showed that a semi-parallel submanifold is the second order envelope of the family of parallel submanifolds [13]. In the case of hypersurfaces in the sphere and the hyperbolic space, F. Dillen showed that they are flat surfaces, hypersurfaces with parallel Weingarten endomorphism or rotation hypersurfaces of certain helices $[9]$.

In [8], the authors obtained some results in hypersurfaces in 4-dimensional space form $N^{4}(c)$ satisfying the curvature condition

$$
\bar{R} \cdot h=L Q(g, h)
$$

The submanifolds satisfying the condition (1.2) are called pseudo-parallel (see [1] and $[2])$.

In [1], Asperti et al. considered the isometric immersions $f: M \longrightarrow \tilde{M}^{n+d}(c)$ of $n$-dimensional Riemannian manifold into $(n+d)$-dimensional real space form $\tilde{M}^{n+d}(c)$ satisfying the curvature condition (1.2). They have shown that if $f$ is pseudo-parallel with $H(p)=0$ and $L_{h}(p)-c \geq 0$ then the point $p$ is a geodesic point of $M$, i.e. the second fundamental form vanishes identically, where $H$ is the mean curvature vector of $M$.

They also showed that a pseudo-parallel hypersurfaces of a space form is either quasi-umbilical or a cyclic of Dupin [2].

The study of complex hypersurfaces was initiated by Smyth [18]. He classified the complete Kaehler-Einstein manifolds which occur as hypersurfaces in complex space forms. The corresponding full local classification was given by Chern [4]. Similar classification under the weaker assumption of parallel Ricci tensor was obtained by Takahashi [19] and Nomizu and Smyth [16]. A classification of the complete Kaehler hypersurfaces of space forms which satisfy the condition $R \cdot R=0$ and a partial classification (the case $c \neq 0$ ) of such hypersurfaces satisfying the condition $R \cdot S=0$ were given by Ryan in [17]. He also classified the complex hypersurfaces of $\mathbb{C}^{n+1}$ having $R \cdot S=0$ and constant scalar curvature.

In [7], Deprez et al. presented a new characterization of complex hyperspheres in complex projective spaces, of complex hypercylinders in complex Euclidean spaces and of complex hyperplanes in complex space forms in terms of the conditions on the tensors $R, S, C$ and $B$, where $B$ is the Bochner tensor which was introduced as a complex version of the Weyl conformal curvature tensor $C$ of a Riemannian manifold [3]. In [23], Yaprak studied pseudosymmetry type curvature conditions on Kaehler hypersurfaces. The submanifolds in a complex space form $\tilde{M}^{m}(c) n \geqslant 2$, of constant holomorphic sectional curvature $4 c$, parallel second fundamental form were classified by H.Naitoh in [15]. S.Maeda [14] studied semi-parallel real hypersurfaces in a complex space form $\tilde{M}^{m}(c)$ for $c>0$ and $n \geqslant 3$. In [12] Lobos and Ortega classify all connected Pseudo-parallel real hypersurfaces in a non-flat complex space form. Then, Yıldız et al. [22] studied $C$-totally real pseudo-parallel submanifolds in Sasakian space forms. 
In the present study, we have generalized their results for the case of $M^{n}$, that is a Kaehlerian submanifold of complex space form $\tilde{M}^{m}(c)$ of holomorphic sectional curvature $c$. We will prove the following:

Theorem 1.1. Let $\tilde{M}^{m}(c)$ be complex $m$-dimensional space form of constant holomorphic sectional curvature $c$ and $M^{n}$ be a complex n-dimensional Kaehlerian submanifold of $\tilde{M}(c)$. If $M^{n}$ is pseudo-parallel and $L n-\frac{1}{2}(n+2) c \geqslant 0$, then $M^{n}$ is totally geodesic.

Also, we study Kaehlerian submanifolds of complex space form with recurrent second fundamental form.

\section{Basic Concepts}

Let $\tilde{M}(c)$ be a non-flat complex space form endowed with the metric $g$ of constant holomorphic sectional curvature $c$. We denote by $\nabla, R, S$ and $\tau$ the Levi-Civita connection, Riemann curvature tensor, the Ricci tensor and scalar curvature of $(M, g)$, respectively. The Ricci operator $\mathcal{S}$ is defined by $g(\mathcal{S} X, Y)=S(X, Y)$, where $X, Y \in \chi(M), \chi(M)$ being Lie algebra of vector fields on $M$. We next define endomorphisms $R(X, Y)$ and $X \wedge_{B} Y$ of $\chi(M)$ by

$$
\begin{gathered}
R(X, Y) Z=\nabla_{X} \nabla_{Y} Z-\nabla_{Y} \nabla_{X} Z-\nabla_{[X, Y]} Z \\
\left(X \wedge_{B} Y\right) Z=B(Y, Z) X-B(X, Z) Y
\end{gathered}
$$

respectively, where $X, Y, Z \in \chi(M)$ and $B$ is a symmetric $(0,2)$-tensor.

The concircular curvature tensor $\tilde{Z}$ in a Riemannian manifold $\left(M^{n}, g\right)$ is defined by

$$
\tilde{Z}(X, Y)=R(X, Y)-\frac{\tau}{n(n-1)}\left(X \wedge_{g} Y\right),
$$

respectively, where $\tau$ is the scalar curvature of $M^{n}$.

Now, for a $(0, k)$-tensor field $T, k \geq 1$ and a $(0,2)$-tensor field $B$ on $(M, g)$ we define the tensor $Q(B, T)$ by

$$
\begin{aligned}
Q(B, T)\left(X_{1}, \ldots, X_{k} ; X, Y\right)= & -T\left(\left(X \wedge_{B} Y\right) X_{1}, X_{2}, \ldots, X_{k}\right) \\
& -\ldots-T\left(X_{1}, \ldots, X_{k-1},\left(X \wedge_{B} Y\right) X_{k}\right)
\end{aligned}
$$

respectively. Putting into the above formula $T=h$ and $B=g$, we obtain the tensor $Q(g, h)$.

Let $f: M^{n} \longrightarrow \tilde{M}^{m}(c)$ be an isometric immersion of an complex $n$-dimensional (of real dimension $2 n$ ) $M$ into complex $m$-dimensional (of real dimension $2 m$ ) space form $\tilde{M}^{m}(c)$. We denote by $\nabla$ and $\widetilde{\nabla}$ the Levi-Civita connections of $M^{n}$ and $\tilde{M}^{m}(c)$, respectively. Then for vector fields $X, Y$ which are tangent to $M^{n}$, the second fundamental form $h$ is given by the formula $h(X, Y)=\widetilde{\nabla}_{X} Y-\nabla_{X} Y$. Furthermore, 
for $\xi \in N\left(M^{n}\right), A_{\xi}: T M \longrightarrow T M$ will denote the Weingarten operator in the $\xi$ direction, $A_{\xi} X=\nabla_{X}^{\perp} \xi-\widetilde{\nabla}_{X} \xi$, where $\nabla^{\perp}$ denotes the normal connection of $M$. The second fundamental form $h$ and $A_{\xi}$ are related by $\widetilde{g}(h(X, Y), \xi)=g\left(A_{\xi} X, Y\right)$, where $g$ is the induced metric of $\widetilde{g}$ for any vector fields $X, Y$ tangent to $M$. The mean curvature vector $H$ of $M$ is defined to be

$$
H=\frac{1}{n} \operatorname{Tr}(h) .
$$

A submanifold $M$ is said to be minimal if $H=0$ identically.

The covariant derivative $\bar{\nabla} h$ of $h$ is defined by

$$
\left(\bar{\nabla}_{X} h\right)(Y, Z)=\nabla_{X}^{\perp}(h(Y, Z))-h\left(\nabla_{X} Y, Z\right)-h\left(Y, \nabla_{X} Z\right),
$$

where, $\bar{\nabla} h$ is a normal bundle valued tensor of type $(0,3)$ and is called the third fundamental form of $M$. The equation of Codazzi implies that $\bar{\nabla} h$ is symmetric hence

$$
\left(\bar{\nabla}_{X} h\right)(Y, Z)=\left(\bar{\nabla}_{Y} h\right)(X, Z)=\left(\bar{\nabla}_{Z} h\right)(X, Y)
$$

Here, $\bar{\nabla}$ is called the van der Waerden-Bortolotti connection of $M$. If $\bar{\nabla} h=0$, then $f$ is called parallel, [10].

The second covariant derivative $\bar{\nabla}^{2} h$ of $h$ is defined by

$$
\begin{aligned}
\left(\bar{\nabla}^{2} h\right)(Z, W, X, Y)= & \left(\bar{\nabla}_{X} \bar{\nabla}_{Y} h\right)(Z, W) \\
= & \nabla_{X}^{\perp}\left(\left(\bar{\nabla}_{Y} h\right)(Z, W)\right)-\left(\bar{\nabla}_{Y} h\right)\left(\nabla_{X} Z, W\right) \\
& -\left(\bar{\nabla}_{X} h\right)\left(Z, \nabla_{Y} W\right)-\left(\bar{\nabla}_{\nabla_{X} Y} h\right)(Z, W) .
\end{aligned}
$$

Then we have

$$
\begin{aligned}
\left(\bar{\nabla}_{X} \bar{\nabla}_{Y} h\right)(Z, W)-\left(\bar{\nabla}_{Y} \bar{\nabla}_{X} h\right)(Z, W)= & (\bar{R}(X, Y) \cdot h)(Z, W) \\
(2.8) & R^{\perp}(X, Y) h(Z, W)-h(R(X, Y) Z, W) \\
& -h(Z, R(X, Y) W) .
\end{aligned}
$$

where $\bar{R}$ is the curvature tensor belonging to the connection $\bar{\nabla}$.

\section{Kaehlerian Submanifolds}

Let $\tilde{M}$ be a Kahlerian manifold of complex dimension $m$ (of real dimension $2 m$ ) with almost complex structure $J$ and with Kahlerian metric $g$. Let $M$ be a complex $n$-dimensional analytic submanifold of $\tilde{M}$, that is, the immersion $f: M \longrightarrow \tilde{M}$ is holomorphic, i.e., $J \cdot f_{*}=f_{*} \cdot J$, where $f_{*}$ is the differential of the immersion $f$ and we denote by the same $J$ the induced complex structure on $M$. Then the Riemannian metric $g$, which will be denoted by the same letter of $\tilde{M}$, induced on $M$ is Hermitian. It is easy to see that the second fundamental form with this Hermitian metric $g$ is the restriction of the second fundamental form of $\tilde{M}$ and 
hence is closed. This show that every complex analytic submanifold $M$ a Kaehlerian manifold $\tilde{M}$ is also a Kaehlerian manifold with respect to the induced structure. We call such a submanifold $M$ of a Kaehlerian manifold $\tilde{M}$ a Kaehlerian submanifold. In the other words, a Kaehlerian submanifold $M$ of a Kaehlerian manifold $\tilde{M}$ is an invariant submanifold under the action of the complex structure $J$ of $\tilde{M}$, i.e., $J T_{x}(M) \subset T_{x}(M)$ for every point $x$ of $M[21]$.

For each plane $p$ in the tangent space $T_{x}(M)$, the sectional curvature $K(p)$ is defined to be $K(p)=R(X, Y, X, Y)=g(R(X, Y) Y, X)$, where $\{X, Y\}$ is an orthonormal basis for $p$. If $p$ is invariant by $J$, then $K(p)$ is called holomorphic sectional curvature by $p$. If $K(p)$ is a constant for all $J$-invariant planes $\mathrm{p}$ in $T_{x}(M)$ and for all points $x \in M$ is called a space of constant holomorphic sectional curvature or a complex space form. Sometimes, a complex space form is defined to be a simply connected complete Kaehlerian manifold of constant holomorphic sectional curvature defined by [21]

$$
\tilde{R}(X, Y) Z=\frac{1}{4} c\{g(X, Z) Y-g(Y, Z) X+g(J X, Z) J Y-g(J Y, Z) J X+2 g(J X, Y) J Z\},
$$

for any vector fields $X, Y$ and $Z$ on $M$. If this space is complete and simply connected, it is well known that it is isometric to

- a complex projective space $\mathbb{C} P^{m}(c)$, if $c>0$;

- the complex Euclidean space $\mathbb{C}^{m}$, if $c=0$;

- a complex hyperbolic space $\mathbb{C} H^{m}$, if $c<0$.

The equations of Gauss and Ricci are

$$
\begin{aligned}
g(R(X, Y) Z, W)= & \frac{1}{4} c[g(Y, Z) g(X, W)-g(X, Z) g(Y, W)+g(J Y, Z) g(J X, W) \\
& -g(J X, Z) g(J Y, W)+2 g(X, J Y) g(J Z, W)] \\
& +g(h(Y, Z), h(X, W))-g(h(X, Z), h(Y, W)),
\end{aligned}
$$

and

$$
g(R(X, Y) U, V)+g\left(\left[A_{V}, A_{U}\right] X, Y\right)=\frac{1}{2} c g(X, J Y) g(J U, V)
$$

respectively. For an orthonormal frame field $\left\{e_{1}, e_{2}, \ldots, e_{n}\right\}$ of $M$, the Ricci tensor $S$ is defined by

$$
S(X, Y)=\sum_{k=1}^{n} g\left(R\left(e_{k}, X\right) Y, e_{k}\right) .
$$

Consequently, by the use of (3.1) the equation (3.3) turns into

$$
S(X, Y)=\frac{1}{2}(n+1) c g(X, Y)-\sum_{i} g\left(h\left(X, e_{i}\right), h\left(Y, e_{i}\right)\right) .
$$


Lemma 3.1. [21] The second fundamental form $h$ of a Kaehlerian submanifold $M$ satisfies

$$
h(J X, Y)=h(X, J Y)=J h(X, Y),
$$

or equivalently

$$
J A_{V} X=-A_{V} J X=A_{J V} X
$$

Proposition 3.1. [21] Any Kaehlerian submanifold $M$ is a minimal submanifold.

Theorem 3.1. [21] Let $M^{n}$ be a Kaehlerian hypersurface of a complex space form $\tilde{M}^{n+1}(c)$. Then the following conditions are equivalent:

(i) The Ricci tensor $S$ of $M^{n}$ is parallel;

(ii) The second fundamental form of $M^{n}$ is parallel;

(iii) $M$ is an Einstein manifold.

\section{Proof of the Theorem 1.1}

Let $M^{n}$ be a complex $n$-dimensional (of real dimensional $2 n$ ) Kaehlerian submanifold with complex structure $J$ of a complex $m$-dimensional (of real dimensional $2 m)$ space form $\tilde{M}^{m}(c)$ of constant holomorphic sectional curvature $c$. Take an orthonormal basis $e_{1}, e_{2}, \ldots, e_{2 n}$ in $T_{X}(M)$ such that $e_{n+t}=J e_{t}(t=1, \ldots, n)$ and an orthonormal basis $v_{1}, \ldots, v_{2 p}$ for $T_{X}(M)^{\perp}$ such that $v_{p+s}=J v_{s}(s=1, \ldots, p)$, where we have put $p=m-n$. Then for $1 \leq i, j \leq n, 1 \leq \alpha \leq p$, the components of the second fundamental form $h$ are given by

$$
h_{i j}^{\alpha}=g\left(h\left(e_{i}, e_{j}\right), e_{\alpha}\right) .
$$

Similarly, the components of the first and the second covariant derivative of $h$ are given by

$$
h_{i j k}^{\alpha}=g\left(\left(\bar{\nabla}_{e_{k}} h\right)\left(e_{i}, e_{j}\right), e_{\alpha}\right)=\bar{\nabla}_{e_{k}} h_{i j}^{\alpha},
$$

and

$$
\begin{aligned}
h_{i j k l}^{\alpha} & =g\left(\left(\bar{\nabla}_{e_{l}} \bar{\nabla}_{e_{k}} h\right)\left(e_{i}, e_{j}\right), e_{\alpha}\right) \\
& =\bar{\nabla}_{e_{l}} h_{i j k}^{\alpha} \\
& =\bar{\nabla}_{e_{l}} \bar{\nabla}_{e_{k}} h_{i j}^{\alpha}
\end{aligned}
$$

respectively.

If $f$ is pseudo-parallel, then by definition, the condition

$$
\bar{R}\left(e_{l}, e_{k}\right) \cdot h=L\left[\left(e_{l} \wedge_{g} e_{k}\right)\right] h
$$


is fulfilled where

$$
\left[\left(e_{l} \wedge_{g} e_{k}\right) h\right]\left(e_{i}, e_{j}\right)=-h\left(\left(e_{l} \wedge_{g} e_{k}\right) e_{i}, e_{j}\right)-h\left(e_{i},\left(e_{l} \wedge_{g} e_{k}\right) e_{j}\right)
$$

for $1 \leq i, j, k, l \leq n$. Substituting (2.2) into (4.5), we get

$$
\begin{aligned}
{\left[\left(e_{l} \wedge_{g} e_{k}\right) h\right]\left(e_{i}, e_{j}\right)=} & -g\left(e_{k}, e_{i}\right) h\left(e_{l}, e_{i}\right)+g\left(e_{l}, e_{i}\right) h\left(e_{k}, e_{i}\right) \\
& -g\left(e_{k}, e_{j}\right) h\left(e_{l}, e_{i}\right)+g\left(e_{l}, e_{j}\right) h\left(e_{k}, e_{i}\right) .
\end{aligned}
$$

By (2.8) we have

$$
\left(\bar{R}\left(e_{l}, e_{k}\right) \cdot h\right)\left(e_{i}, e_{j}\right)=\left(\bar{\nabla}_{e_{l}} \bar{\nabla}_{e_{k}} h\right)\left(e_{i}, e_{j}\right)-\left(\bar{\nabla}_{e_{k}} \bar{\nabla}_{e_{l}} h\right)\left(e_{i}, e_{j}\right) .
$$

Making use of (4.1), (4.3), (4.6) and (4.7), the pseudo-parallelity condition (4.4) turns into

$$
h_{i j k l}^{\alpha}=h_{i j l k}^{\alpha}-L\left\{\delta_{k i} h_{l j}^{\alpha}-\delta_{l i} h_{k j}^{\alpha}+\delta_{k j} h_{i l}^{\alpha}-\delta_{l j} h_{k i}^{\alpha}\right\},
$$

where $g\left(e_{i}, e_{j}\right)=\delta_{i j}$ and $1 \leq i, j, k, l \leq n, 1 \leq \alpha \leq p$.

Recall that the Laplacian $\Delta h_{i j}^{\alpha}$ of $h_{i j}^{\alpha}$ is defined by

$$
\Delta h_{i j}^{\alpha}=\sum_{i, j, k=1}^{n} h_{i j k k}^{\alpha}
$$

Then we obtain

$$
\frac{1}{2} \Delta\left(\|h\|^{2}\right)=\sum_{i, j, k=1}^{n} \sum_{\alpha=1}^{p} h_{i j}^{\alpha} h_{i j k k}^{\alpha}+\|\bar{\nabla} h\|^{2},
$$

where

$$
\|h\|^{2}=\sum_{i, j, k=1}^{n} \sum_{\alpha=1}^{p}\left(h_{i j}^{\alpha}\right)^{2}
$$

and

$$
\|\bar{\nabla} h\|^{2}=\sum_{i, j, k=1}^{n} \sum_{\alpha=1}^{p}\left(h_{i j k k}^{\alpha}\right)^{2}
$$

are the square of the length of second and the third fundamental forms of $M^{n}$, respectively. In addition, making use of (4.1) and (4.3), we obtain

$$
\begin{aligned}
h_{i j}^{\alpha} h_{i j k k}^{\alpha} & =g\left(h\left(e_{i}, e_{j}\right), e_{\alpha}\right) g\left(\left(\bar{\nabla}_{e_{k}} \bar{\nabla}_{e_{k}} h\right)\left(e_{i}, e_{j}\right), e_{\alpha}\right) \\
& =g\left(\left(\bar{\nabla}_{e_{k}} \bar{\nabla}_{e_{k}} h\right)\left(e_{i}, e_{j}\right) g\left(h\left(e_{i}, e_{j}\right), e_{\alpha}\right), e_{\alpha}\right) \\
& =g\left(\left(\bar{\nabla}_{e_{k}} \bar{\nabla}_{e_{k}} h\right)\left(e_{i}, e_{j}\right), h\left(e_{i}, e_{j}\right)\right) .
\end{aligned}
$$

Therefore, due to (4.13), the equation (4.10) becomes

$$
\frac{1}{2} \Delta\left(\|h\|^{2}\right)=\sum_{i, j, k=1}^{n} g\left(\left(\bar{\nabla}_{e_{k}} \bar{\nabla}_{e_{k}} h\right)\left(e_{i}, e_{j}\right), h\left(e_{i}, e_{j}\right)\right)+\|\bar{\nabla} h\|^{2} .
$$


Further, by the use of (4.4), (4.6) and (4.7), we get

$$
\begin{aligned}
g\left(\left(\bar{\nabla}_{e_{k}} \bar{\nabla}_{e_{k}} h\right)\left(e_{i}, e_{j}\right), h\left(e_{i}, e_{j}\right)=\right. & g\left(\left(\bar{\nabla}_{e_{k}} \bar{\nabla}_{e_{i}} h\right)\left(e_{k}, e_{j}\right), h\left(e_{i}, e_{j}\right)\right) \\
= & g\left(\left(\bar{\nabla}_{e_{i}} \bar{\nabla}_{e_{k}} h\right)\left(e_{j}, e_{k}\right), h\left(e_{i}, e_{j}\right)\right) \\
& -L\left\{g\left(e_{i}, e_{j}\right) g\left(h\left(e_{k}, e_{k}\right), h\left(e_{i}, e_{j}\right)\right)\right. \\
& -g\left(e_{k}, e_{j}\right) g\left(h\left(e_{k}, e_{i}\right), h\left(e_{i}, e_{j}\right)\right) \\
& +g\left(e_{k}, e_{i}\right) g\left(h\left(e_{j}, e_{k}\right), h\left(e_{i}, e_{j}\right)\right) \\
& \left.-g\left(e_{k}, e_{k}\right) g\left(h\left(e_{i}, e_{j}\right), h\left(e_{i}, e_{j}\right)\right)\right\} .
\end{aligned}
$$

Substituting (4.15) into (4.14), we have

$$
\begin{aligned}
\frac{1}{2} \Delta\left(\|h\|^{2}\right)= & \sum_{i, j, k=1}^{n}\left[g\left(\left(\bar{\nabla}_{e_{i}} \bar{\nabla}_{e_{j}} h\right)\left(e_{k}, e_{k}\right), h\left(e_{i}, e_{j}\right)\right)\right. \\
& -L\left\{g\left(e_{i}, e_{j}\right) g\left(h\left(e_{k}, e_{k}\right), h\left(e_{i}, e_{j}\right)\right)\right. \\
& -g\left(e_{k}, e_{j}\right) g\left(h\left(e_{k}, e_{i}\right), h\left(e_{i}, e_{j}\right)\right) \\
& +g\left(e_{k}, e_{i}\right) g\left(h\left(e_{j}, e_{k}\right), h\left(e_{i}, e_{j}\right)\right) \\
& \left.\left.-g\left(e_{k}, e_{k}\right) g\left(h\left(e_{i}, e_{j}\right), h\left(e_{i}, e_{j}\right)\right)\right\}\right]+\|\bar{\nabla} h\|^{2}
\end{aligned}
$$

Furthermore, by definition

$$
\begin{gathered}
\|h\|^{2}=\sum_{i, j=1}^{n} g\left(h\left(e_{i}, e_{j}\right), h\left(e_{i}, e_{j}\right)\right), \\
H^{\alpha}=\sum_{k=1}^{n} h_{k k}^{\alpha}, \\
\|H\|^{2}=\frac{1}{n^{2}} \sum_{\alpha=1}^{p}\left(H^{\alpha}\right)^{2}
\end{gathered}
$$

and after some calculations, we get

$$
\begin{aligned}
\frac{1}{2} \Delta\left(\|h\|^{2}\right)= & \sum_{i, j=1}^{n} \sum_{\alpha=1}^{p} h_{i j}^{\alpha}\left(\bar{\nabla}_{e_{i}} \bar{\nabla}_{e_{j}} H^{\alpha}\right) \\
& -L\left\{n^{2}\|H\|^{2}-n\|h\|^{2}\right\}+\|\bar{\nabla} h\|^{2} .
\end{aligned}
$$

Using Proposition 3.1, the equation (4.18) is reduced to

$$
\frac{1}{2} \Delta\left(\|h\|^{2}\right)=L n\|h\|^{2}+\|\bar{\nabla} h\|^{2} .
$$


Yano and Kon have shown in [21], that

$$
\begin{aligned}
\frac{1}{2} \Delta\left(\|h\|^{2}\right)= & \|\bar{\nabla} h\|^{2}-\sum_{\alpha, \beta=1}^{p}\left\{\left[\operatorname{Tr}\left(A_{\alpha} \circ A_{\beta}\right)\right]^{2}+\left\|\left[A_{\alpha}, A_{\beta}\right]\right\|^{2}\right. \\
& +\frac{1}{2}(n+2) c\|h\|^{2} .
\end{aligned}
$$

Hence comparing the equation (4.19) with (4.20), one can get

$$
\begin{aligned}
0= & \left(\text { Ln }-\frac{1}{2}(n+2) c\right)\|h\|^{2} \\
& +\sum_{\alpha, \beta=1}^{p}\left\{\left[\operatorname{Tr}\left(A_{\alpha} \circ A_{\beta}\right)\right]^{2}+\left\|\left[A_{\alpha}, A_{\beta}\right]\right\|^{2}\right\} .
\end{aligned}
$$

If $L n-\frac{1}{2}(n+2) c \geq 0$ then $\operatorname{Tr}\left(A_{\alpha} \circ A_{\beta}\right)=0$. In particular, $\left\|A_{\alpha}\right\|^{2}=\operatorname{Tr}\left(A_{\alpha} \circ\right.$ $\left.A_{\alpha}\right)=0$, hence $h=0$. This completes the proof of our Theorem.

Corollary 4.1. Let $\tilde{M}^{m}(c)$ be complex m-dimensional space form of constant holomorphic sectional curvature $c$ and $M^{n}$ be a complex n-dimensional Kaehlerian submanifold of $\tilde{M}^{m}(c)$. If $\tilde{Z}(X, Y) \cdot h=0$ and $\frac{\tau}{(n-1)}-\frac{1}{2}(n+2) c \geq 0$ then $M$ is totally geodesic.

We recall the following well-known:

Theorem 4.1. ([4], [16], [19]) Let $M^{n}$ be a Kaehlerian hypersurface of a complex space form $\tilde{M}^{n+1}(c)$ with parallel Ricci tensor. If $c \leq 0$, then $M^{n}$ is totally geodesic. If $c>0$, then either $M$ is totally geodesic, or an Einstein manifold $|A|^{2}=n c$ and hence $\tau=n^{2} c$.

Using Theorem 3.1 and Theorem 4.1, we can easily obtain the following:

Corollary 4.2. Let $M^{n}$ be a Kaehlerian hypersurface of a complex space form $\tilde{M}^{n+1}(c)$ with parallel second fundamental form. If $c \leq 0$, then $M^{n}$ is totally geodesic.

Using Theorem 1.1, we get the following:

Corollary 4.3. Let $M^{n}$ be a complex n-dimensional Kaehlerian submanifold of $\tilde{M}(c)$ with semi-parallel. If $c \leq 0$, then $M^{n}$ is totally geodesic.

Remark 4.1. (i) The main Theorem is generalization of Corollary 4.2 and Corollary 4.3. (ii) If second fundamental form of $M^{n}$ is parallel then it is semi-parallel. But the converse is not necessary to be parallel. 


\section{Kaehlerian submanifolds of comlex space form with recurrent second fundamental form}

In this section, we will consider the condition by which the second fundamental tensor $A$ is recurrent, i.e., there exists a 1 -form $\alpha$ such that $\bar{\nabla} A=\alpha \otimes A$. We may regard parallel condition as a special case. We know that the recurrent condition has a close relation to a holonomy group (cf,[11], [20]). Using definition of recurrent second fundamental form, we get

$$
\bar{\nabla}_{X} \bar{\nabla}_{Y} A=(X \alpha(Y)+\alpha(X) \alpha(Y)) A,
$$

which implies that

$$
\begin{aligned}
\bar{R}(X, Y) \cdot A= & \bar{\nabla}_{X} \bar{\nabla}_{Y} A-\bar{\nabla}_{Y} \bar{\nabla}_{X} A-\bar{\nabla}_{[X, Y]} A \\
= & (X \alpha(Y)+\alpha(X) \alpha(Y)) A-(Y \alpha(X)+\alpha(Y) \alpha(X)) A \\
& -\alpha([X, Y]) A \\
= & (X \alpha(Y)-Y \alpha(X)-\alpha([X, Y]) A \\
= & 2 d \alpha(X, Y) A .
\end{aligned}
$$

We now define a function on $M^{n}$ by $f^{2}=g(A, A)$, where the metric $g$ is extended to the inner product between the tensor fields in the standard fashion [11]. Using the fact that $\nabla g=0$ it follows from $f^{2}=g(A, A)$ that

$$
f(Y(f))=f^{2} \alpha(Y) .
$$

So from (5.2), we have

$$
Y f=f \alpha(Y) \neq 0
$$

Therefore we get

$$
\left\{\bar{\nabla}_{X} \bar{\nabla}_{Y}-\bar{\nabla}_{Y} \bar{\nabla}_{X}-\bar{\nabla}_{[X, Y]}\right\} f=\{X \alpha(Y)-Y \alpha(X)-\alpha([X, Y]\} f .
$$

Since the left hand side of the above equation is identically zero and $f \neq 0$ on $M$ by our assumption, we obtain

$$
d \alpha(X, Y)=0,
$$

that is, the 1-form $\alpha$ is closed. Hence, from (5.1) and (5.5), we get

$$
\bar{R}(X, Y) \cdot A=0 .
$$

It means that $M$ is semi-parallel. So, by the use of Corollary 4.3 , we can give the following:

Theorem 5.1. Let $\tilde{M}^{m}(c)$ be complex m-dimensional space form of constant holomorphic sectional curvature $c$. If $c \leq 0$, there are no Kaehler submanifolds with non-trivial recurrent second fundamental form of $\tilde{M}^{m}(c)$. 


\section{REFEREN CES}

1. A. C. Asperti, G. A. Lobos and F. Mercuri: Pseudo-paralel immersions in space forms. Math. Contemp. 17 (1999), 59-70.

2. A. C. Asperti, G. A. Lobos and F. Mercuri: Pseudo-parallel immersions of a space forms. Adv. Geom. 2 (2002), 57-71.

3. S. Bochner: Curvature and Betti numbers, II. Ann. Math. 50 (1949), 77-94.

4. S. S. CHERN: Einstein hypersurfaces in a Kaehler manifold of constant holomorphic curvature. J. Diff. Geo. 1 (1967), 21-31.

5. J. Deprez: Semi-Parallel Surfaces in Euclidean space. J. Geom. 25 (1985), 192200.

6. J. Deprez: Semi-Parallel Hypersurfaces. Rend. Sem. Mat. Univers. Politecn. Torino. 44 (1986), 303-316.

7. J. Deprez, P. Verheyen and L. Verstraelen: Intrinsic characterizations for complex hypercylinders and complex hyperspheres. Geom. Dedicata. 16 (1984), $217-229$.

8. R. Deszcz, L. Verstraelen and S. Yaprak: Pseudosymmetric hypersurfaces in 4-dimensional space of constant curvature. Bull. Ins. Math. Acad. Sinica. 22 (1994), 167-179.

9. F. Dillen: Semi-parallel hypersurfaces of a real space form. Israel J. Math. 75 (1991), 193-202.

10. D. Ferus: Immersions with parallel second fundamental form. Math. Z. 140 (1974), 87-93.

11. S. Kobayashi and K. Nomizu: Foundations of Differential Geometry Volume I. A Willey Interscience Publication John Willey \& Sons, 1963.

12. G. A. Lobos and M. Ortega: Pseudo-parallel real hypersurface in complex space forms. Bull. Korean Math. Soc. 41 (2004), 609-618.

13. Ü. LUMISTE: Semi-symmetric submanifolds as the second order envelope of symmetric submanifolds. Proc. Estonian Acad. Sci. Phys. Math. 39 (1990), 1-8.

14. S. Maeda: Real hypersurfaces of a complex projective spaces. Math. Ann. 263 (1983), 473-478.

15. H. NAitoh: Parallel submanifolds of complex space forms I and II. Nagoya Math. J. I 90 (1983), 85-117, II 91 (1983), 119-149.

16. K. Nomizu and B. Sмүтн: Differential geometry of complex hypersurfaces, II. J. Math. Society of Japan. 20 (1968), 498-521.

17. P. J. Ryan: A class of complex hypersurfaces. Colloqium Math. J. 26 (1972), $175-182$.

18. J. Sмүтн: Homogeneous complex hypersurfaces. J. Math. Society of Japan. 20 (1968), 643-647.

19. T. TAKAHASHI: Hypersurfaces with parallel Ricci tensor in a space of constant holomorphic sectional curvature. J. Math. Society of Japan. 19 (1967), 199-204.

20. Y. C. WONG: Recurrent tensors on a linearly connected differentiable manifold. Trans. Amer. Math. Soc. 99 (1961), 325-341.

21. K. YANO and M. Kon: Structures on manifolds. World Scientific. (1984), 508p. 
22. A. Yildiz, C. Murathan, K. Arslan and R. Ezentaş: C-totally real pseudo parallel submanifolds of Sasakian space forms. Monatshefte für Mathematik. 151(3) (2007), 247-256.

23. S. YAPRAK: Pseudosymmetry type curvature conditions on Kaehler hypersurfaces. Math.J. Toyama Univ. 18 (1995), 107-136.
Ahmet Yildız
Education Faculty
Department of Mathematics
P.O. Box 44280
Malatya, Turkey
a.yildiz@inonu.edu.tr 